\title{
Distribution of dominant arbuscular mycorrhizal fungi among five plant species in undisturbed vegetation of a coastal grassland
}

\section{Journal Article}

Author(s):

Stukenbrock, Eva H.; Rosendahl, Søren

Publication date:

2005-11

Permanent link:

https://doi.org/10.3929/ethz-b-000031179

Rights / license:

In Copyright - Non-Commercial Use Permitted

Originally published in:

Mycorrhiza 15(7), https://doi.org/10.1007/s00572-005-0357-2 


\section{Eva H. Stukenbrock • Søren Rosendahl \\ Distribution of dominant arbuscular mycorrhizal fungi among five plant species in undisturbed vegetation of a coastal grassland}

Received: 5 October 2004 / Accepted: 25 February 2005 / Published online: 5 April 2005

(C) Springer-Verlag 2005

\begin{abstract}
Most plant species in mixed grassland vegetation are colonized by arbuscular mycorrhizal (AM) fungi. Previous studies have reported differences in host preferences among AM fungi, although the fungi are known to lack host specificity. In the present study, the distribution of phylogenetic groups of AM fungi belonging to a clade of Glomus species was studied in five plant species from a coastal grassland in Denmark. The occurrence of the fungi was determined by PCR analyses of fungal large subunit ribosomal DNA sequences amplified from root fragments using a specific primer set. The results showed that the dominant Glomus species were able to colonize all the studied plant species, supporting the view that the AM fungi represent a large underground interconnecting mycelial network.
\end{abstract}

Keywords Arbuscular mycorrhizal fungi - Community composition · Host plant preferences · Mycorrhizal networks $\cdot$ Spatial distribution

\section{Introduction}

The fact that most arbuscular mycorrhizal (AM) fungi can colonize a variety of plant species indicates an absence of host specificity (Mosse 1975). This has been demonstrated by Helgason et al. (1998), who found identical sequences of AM fungi in roots of different host plant species in both woodland and agricultural systems, suggesting that the same fungi were colonizing different plants. Lack of host pref-

\footnotetext{
E. H. Stukenbrock $(\bowtie) \cdot S$. Rosendahl

Department of Microbiology, Biological Institute,

University of Copenhagen,

Øster Farimagsgade 2D,

1353 Copenhagen K., Denmark

e-mail: eva.stukenbrock@ipw.agrl.ethz.ch

Present address:

E. H. Stukenbrock

Institute for Plant Sciences, Phytopathology Group,

Federal Institute of Technology,

ETH-Zentrum, LWF, Universitätstrasse 2,

8092 Zurich, Switzerland
}

erences among AM fungi is supported by several observations showing that root systems of different plant species and with different photosynthetic capacity can be interconnected through common mycelia networks (Birch 1986; Grime et al. 1987; Giovannetti et al. 2004). Seedlings growing up in a mycorrhizal community can in this way rapidly become linked into, and acquire nutrients from, a mycelial network that has developed at the expense of photosynthate from already established plants.

Other studies have, however, questioned the random colonization of different host plant species. Non-random associations between AM fungi and different host species have been demonstrated in other studies, suggesting some degree of fungal host preferences (Husband et al. 2002; Vandenkoornhuyse et al. 2002; Gollotte et al. 2004). Helgason et al. (2002) found that the fungal community structure on roots of five woodland plant species was determined by the different hosts. Isolation of native fungi and establishment of one-to-one AM symbioses showed that colonization pattern, symbiont compatibility and plant performance varied with the plant-fungus combination. Inter- and intraspecific functional differences between fungal species have been demonstrated and can be responsible for the observed variation in plant-fungus compatibility (Smith et al. 2000; Koch et al. 2004; Munkvold et al. 2004).

The conflicting conclusions regarding host preferences may be due to results obtained at different phylogenetic levels. The formation of mycelial networks has been demonstrated for single AM species or isolates (Grime et al. 1987; Giovannetti et al. 2004), whereas a non-random distribution in the field has been demonstrated between AM fungi of different species and genera (Helgason et al. 2002; Husband et al. 2002; Gollotte et al. 2004). Mycelial networks may be formed only between closely related fungi and may be characteristic only for some species or only in some vegetation systems.

In molecular studies of fungal distribution, the selection of primers and the amount of genetic variation in the target sequence determine the phylogenetic levels that are covered. Kjøller and Rosendahl (2000) have developed a set of specific primers targeting a clade of Glomus species 
including G. mosseae, G. caledonium, G. geosporum and G. intraradices. The primer set amplifies a 300-bp sequence of the D2 region in the large subunit ribosomal DNA (LSU rDNA). Amplification of Glomus sequences from colonized plant roots has been used to study community structure of this particular clade in agricultural systems (Kjøller and Rosendahl 2000) and in submerged plants (Nielsen et al. 2004). Rosendahl and Stukenbrock (2004) used the primers in a nested PCR approach to characterize the community of Glomus species colonizing Hieracium pilosella in a coastal grassland. It was found that roots of $H$. pilosella were dominated by unknown phylogenetic groups that were not present in the soil as spores. The dominant fungi showed non-uniform distributions along a $30-\mathrm{m}$ transect, and it is possible that the spatial structuring of these Glomus species reflects the presence of belowground mycelial networks. Because only mycorrhizal fungi in roots of $H$. pilosella were identified, the aim of the present study was to clarify if these dominating phylogenetic clusters were also colonizing other abundant plant species along the same transect.

\section{Materials and methods}

\section{Sampling}

The studied site is a coastal undisturbed sandy grassland on the north coast of Zeeland, Denmark. The soil has a low nutrient content with a $\mathrm{pH}$ from 6.5 to 7.5 . The plant community is characterized by high species diversity dominated by perennial herbs (Rosendahl and Stukenbrock 2004). Five plant species were chosen on basis of their high abundance at the field site: H. pilosella, Hypochoeris radicata, Thymus serpyllum, Artemisia campestris and Armeria maritima. Plants were harvested in late May and beginning of July 2002. Six plots were set up at a 30-m transect line perpendicular to the coastline, with a distance of approximately $5 \mathrm{~m}$ between each plot. Plants of the five host species growing together in a square of $20 \times 20 \mathrm{~cm}$ were collected.

Two plants of each species from each harvest were used. Roots were washed carefully, eight pieces of approximately $0.5 \mathrm{~cm}$ were cut with a razor blade from each plant and frozen in $0.5-\mathrm{ml}$ Eppendorf tubes in $60 \mu \mathrm{l} \mathrm{TE}$ buffer for later analysis. In order to determine the degree of mycorrhizal colonization, roots were cleared in $10 \% \mathrm{KOH}$ for $24 \mathrm{~h}$ at room temperature. The cleared root pieces were rinsed in $1 \% \mathrm{HCl}$ and then stained with $0.05 \%$ Trypan blue for $1 \mathrm{~h}$ at $60^{\circ} \mathrm{C}$ (Phillips and Hayman 1970). After washing in water, roots were stored in lactoglycerol. The mycorrhizal colonization was determined with the line-intersect method using a light microscope (Giovannetti and Mosse 1980).

\section{DNA extraction and PCR}

Root pieces were crushed in 0.5-ml Eppendorf tubes in TE buffer using plastic pistilles. For DNA extraction, samples were denatured for $2 \mathrm{~min}$ at $95^{\circ} \mathrm{C}$ in the presence of $20 \mu \mathrm{l}$ 20\% Chelex-100 (BioRad). Samples were vortexed and centrifuged at $10,000 \times \mathrm{g}, 4^{\circ} \mathrm{C}$ for $5 \mathrm{~min}$. Five microliters supernatant was recovered and diluted 100 times in $500 \mu \mathrm{l}$ sterilised water.

The primary amplification was performed in a final volume of approximately $20 \mu \mathrm{l}$ containing $2 \mu \mathrm{l}$ template and a master mix of $2 \mu \mathrm{l}$ TQ buffer [167.5 mM Tris/ $\mathrm{HCl}(\mathrm{pH} 8.5)$, $5 \mathrm{mM}\left(\mathrm{NH}_{4}\right) \mathrm{SO}_{4}$ and $25 \mathrm{mM} \beta$-mercaptoethanol], $2 \mu \mathrm{l}$ of each eucaryotic primer, 0061f and NDL22r (van Tuinen et al. 1998a), $5 \mu \mathrm{l}$ sterilised $\mathrm{H}_{2} \mathrm{O}, 8 \mu \mathrm{l}$ GATC mix and $0.1 \mu \mathrm{l}$ Taq polymerase (Amersham, Bioscience). Amplification was performed in a thermocycler (PCT-200, MJ Research Inc., Watertown, MA) with cycling as described by Kjøller and Rosendahl (2000).

Primary PCR products were diluted 100 times. Two microliters of the diluted PCR products were used as template in nested PCR with the specific primers LSURK4f and LSURK $7 \mathrm{mr}$. These primers allow amplification of a clade of Glomus species including G. mosseae, G. caledonium, $G$. geosporum, $G$. coronatum, G. fragilistratum and $G$. constrictum (Kjøller and Rosendahl 2000; Rosendahl and Stukenbrock 2004). Amplification cycles for nested PCR reaction were as described by Kjøller and Rosendahl (2000). PCR products were checked on agarose gels containing $2 \%$ Nusieve in 1\% TE buffer and stained with ethidium bromide.

Positive PCR products were purified using the QIAquick PCR purification kit protocol from Qiagen. Sequencing of PCR products was performed by MWG Biotech (Ebersberg, Germany) using LSURK4f as sequencing primer.

The sequences were aligned manually and edited using the program Bioedit Sequence Alignment Editor (http://www. mbio.ncsu.edu/BioEdit/bioedit.html). Sequences from registered BEG isolates were included in the alignment except G. microaggregatum and G. sinuosum that originated from environmental samples. Sequences of the phylogenetic clusters defined by Rosendahl and Stukenbrock (2004) were also included. The phylogeny of the sequences was analyzed using maximum parsimony and maximum likelihood. All analyses were conducted with PAUP4.0b. All characters were equally weighted and alignment gaps were treated as missing data. Parsimony analyses consisted of heuristic searches with 1,000 random addition sequences and tree bisection-reconnection (TBR) branch swapping. Maximum likelihood (ML) was performed on the combined data set after the exclusion of all identical taxa. Supports for the internal nodes of the most parsimonious tree (MPT) were assessed by 1,000 bootstrap replications. The resulting trees were used to infer phylogenetic clusters, defined as separate phylogenetic lineages with high bootstraps and which appeared in both the ML tree and the MPT.

The null hypothesis that there were no differences in the distribution of the phylogenetic clusters among the plants was tested using the Kruskal-Wallis test and by ranking the observed frequencies of the phylogenetic clusters.

\section{Results}

Plants were heavily colonized by mycorrhizal fungi, except for A. maritima in which mycorrhizal colonization was less 
than for the other plant species (Table 1). Morphological structures of different AM fungi, fine endophytes and unidentified root endophytes were recognized in the stained roots; however, it was not possible to identify any of the AM fungi at species level.

LSU rDNA sequences were successfully amplified by the non-specific eucaryotic primers by primary PCR. Glomus sequences ( $300 \mathrm{bp}$ ) were amplified in nested PCR with the primer set LSURK4f and LSURK7mr. Sequencing of nested PCR products gave 91 unique sequences. Results from nested PCR differed between the five plant species (Table 1): $24.7-37.4 \%$ positive PCRs were obtained from roots of $H$. pilosella, $H$. radicata and $T$. serpyllum, whereas the percentage of positive nested PCRs was significant lower in A. campestris and A. maritima.

The maximum likelihood analysis separated the Glomus sequences into eight phylogenetic clusters (Fig. 1). The phylogenetic cluster D formed the most common group. The other dominant phylogenetic cluster was composed of the subgroups $\mathrm{F}$ and $\mathrm{G}$. Most sequences within these unidentified phylogenetic clusters differed by only one or a few base pairs. Only a few of the detected sequences could be associated to taxonomically described species: $G$. intraradices, G. caledonium, G. mosseae and G. microaggregatum.

No variation in sequence distribution was found between the two harvests. Sequences from both harvest times were therefore pooled for further analyses. The dominant clusters $\mathrm{D}$ and $\mathrm{F}+\mathrm{G}$ were found in roots of all five plant species (Figs. 1 and 2). G. mosseae sequences were similarly detected in roots of all host species except $A$. maritima. The other phylogenetic clusters were only rarely detected. Phylogenetic clusters were randomly distributed among four host plant species (Fig. 2), and the Kruskal-Wallis test on the ranked data set gave a $K$ value of 0.8 , indicating no significant difference between the frequencies of the phylogenetic clusters in these four plant species. A. maritima was not included in the test because too few observations were made in this species.

The cluster D was present in plant roots from all plots along the transect; however, the frequency of the cluster varied between the plots (Fig. 3). The other dominant group, $\mathrm{F}+\mathrm{G}$, was present in three plots, and was most common in the 20 -m plot. G. mosseae could only be detected in the 32 and 40-m plots (Fig. 3).

\section{Discussion}

The dominating phylogenetic cluster $\mathrm{D}$, obtained from the different plant species, belong to the same unknown Glo$m u s$ cluster that was previously found to be dominant in roots of $H$. pilosella along the same transect (Rosendahl and Stukenbrock 2004). In the study by Rosendahl and Stukenbrock (2004), wherein additional clusters were also identified on the roots of $H$. pilosella using the same approach, sampling was much more intense along eight transects, allowing detection of less frequent fungi not found in this study.

We found a discrepancy between the degree of colonization within plant roots and successful nested PCR amplification of the Glomus LSU rDNA sequences. This discrepency may be explained by the presence of other genera of AM fungi not targeted by the specific Glomus primers. Other community studies of AM fungi have used primers that amplify less specific sequences in the small subunit (SSU) rDNA thereby allowing the detection of a broader diversity (Helgason et al. 1998; Husband et al. 2002; Kowalchuk et al. 2002). However, one disadvantage of using SSU rDNA sequences is that the low extent of genetic variability in this gene only allows identification of AM fungi above species level (van Tuinen et al. 1998b). Because we were interested in the distribution of one particular clade of Glomus fungi, sequences with a higher resolution of genetic variability were targeted.

In other AM fungal community studies wherein qualitative data have been obtained by cloning PCR products obtained from whole root systems (van Tuinen et al. 1998b; Daniell et al. 2001; Helgason et al. 2002), quantitative information is lost. In the previous study by Rosendahl and Stukenbrock (2004), cloning was initially performed on amplicons of nested PCR products. However, because no new sequences were revealed by cloning, sequencing was performed directly on nested PCR products. The occurrence of multiple sequences or sequence chimeras in the PCR products were not detected in the sequence chromatograms. Polymorphisms in the LSU sequences were found at the same sites, which additionally indicates that the observed variation was a result of true mutations and not PCR artefacts. Nested PCR on eight root fragments from each root system was therefore applied instead of cloning. This permitted assessment of frequencies of the amplified Glomus

Table 1 Mycorrhizal colonization and success rate of PCR amplification from plant roots of the five host plant species

\begin{tabular}{llllcc}
\hline Plant species & \% Colonization & No. of root fragments & Positive primary PCR & Positive nested PCR & \% Positive nested PCR \pm SE \\
\hline H. pilosella & $83(8)^{*}$ & 160 & 156 & 49 & $31.4 \pm 6.3$ \\
H. radicata & $85(8)$ & 192 & 190 & 71 & $37.4 \pm 8.0$ \\
T. serpyllum & $77(3)$ & 192 & 174 & 43 & $24.7 \pm 4.4$ \\
A. maritima & $40(4)$ & 136 & 112 & 5 & $4.5 \pm 2.4$ \\
A. campestris & $88(1)$ & 132 & 114 & 186 & $15.8 \pm 3.7$ \\
Total & & 812 & 746 & & 22.7 \\
\hline
\end{tabular}

*Number of root systems examined by the line intersect method

Positive nested PCRs were estimated from the percentage positive primary PCRs giving products 


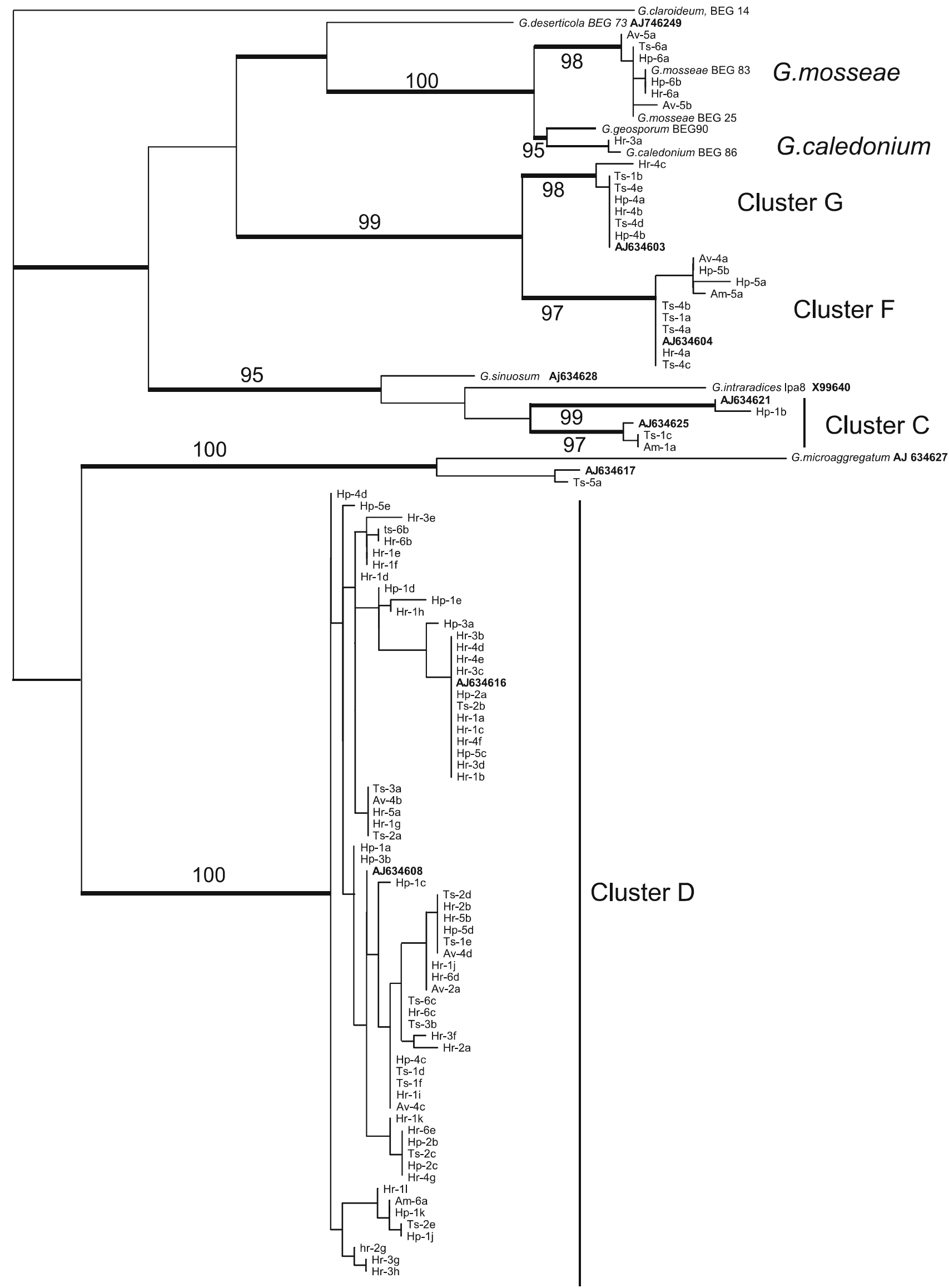


Fig. 1 Phylogenetic analysis using maximum likelihood of the Glomus sequences obtained from roots of $H$. pilosella $(H p), H$. radicata $(\mathrm{Hr})$, T. serpyllum $(\mathrm{Ts})$, A. campestris $(A c)$ and A. maritima (Am). Numbers indicate plot numbers along the transect (1-6). Different plants of the same species and same plot are distinguished by different letters $(a-c)$. Bootstrap values above 90 are indicated. The monophyletic clades interpreted as phylogenetic clusters are indicated. Species names are derived from sequences obtained from known BEG registered cultures, except $G$. sinuosum and $G$. microaggregatum, which were obtained from field samples. Sequences in the phylogenetic clusters $\mathrm{C}, \mathrm{D}, \mathrm{F}$ and $\mathrm{G}$, derived from the previous study of $\mathrm{H}$. pilosella, are indicated by their respective accession numbers $(A J)$

sequences and thereby to determine the quantitative diversity of the phylogenetic clusters present in the plant roots.

The different frequencies of the dominant sequence types between plots and host plants suggest a patchy distribution of the Glomus species, similar to the distribution of phylogenetic clusters in the study by Rosendahl and Stukenbrock (2004). Patchy distributions of ectomycorrhizal commu- nities at a fine scale have also been demonstrated in several studies (Horton and Bruns 2001), and similar patterns may be characteristic for AM fungal communities. The factors responsible for the patchy distribution of the Glomus sequences are unknown. Several of the detected Glomus species may have no or only limited spore production and the sequence types may represent clones of Glomus fungi derived from hyphal propagation (Rosendahl and Stukenbrock 2004). If horizontal spread of AM fungal clones is restricted by soil conditions, it is possible that host preferences of the fungi will remain less specific at an undisturbed site in order to maintain the ability of the fungi to colonize different plants growing closely together. The random association between the dominant mycorrhizal fungi and different host plants support the hypothesis of an interconnecting mycelial network.

The observed random association between host plant species and the dominant phylogenetic Glomus species is inconsistent with other studies of AM fungal host pref-
Fig. 2 Distribution of the phylogenetic clusters $\mathrm{D}, \mathrm{F}+\mathrm{G}$ and four Glomus species present in the roots of the four host plant species indicated as the frequency of each fungal species in each plant species.

A. maritima was not included as only three sequences were obtained. G. mos, G. mosseae; G. micro, G. microaggregatum; $G$. intra, $G$. intraradices; $G$. caled, $G$. caledonium

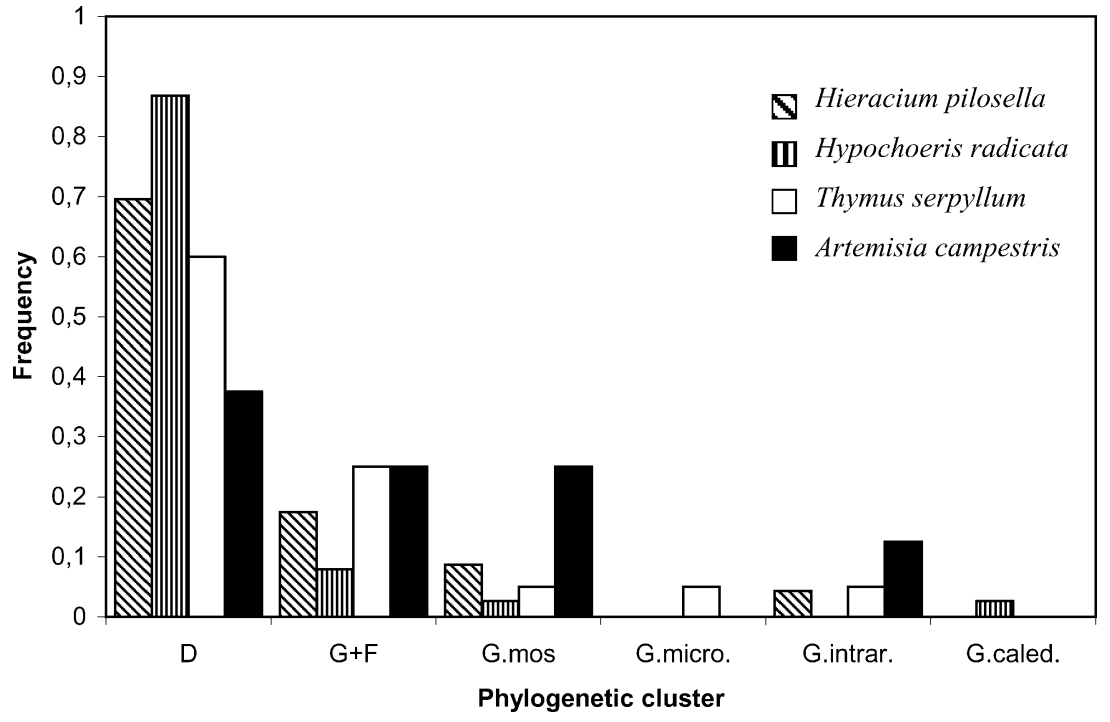

Fig. 3 Distribution in six plots along the transect of the coastal grassland of the phylogenetic clusters $\mathrm{D}$ and $\mathrm{F}+\mathrm{G}, \mathrm{G}$. mosseae and a group including $G$. caledonium, $G$. intraradices and G. microaggregatum (other)

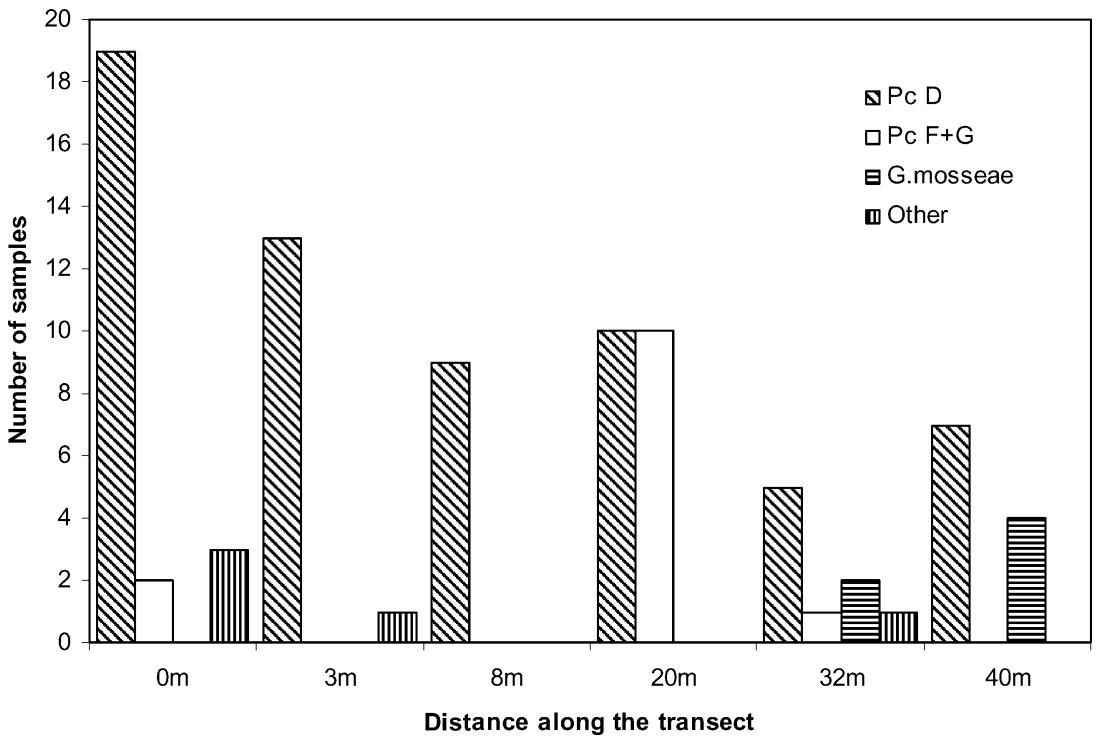


erences, as some degree of host preferences has been shown to exist in certain ecosystems between different genera of AM fungi and different host plant species (Helgason et al. 1998; Husband et al. 2002; Kowalchuk et al. 2002). In a study at a finer phylogenetic level, Gollotte et al. (2004) used a set of LSU rDNA taxon-discriminating primers to demonstrate the presence of different fungal communities in roots of two grass species in an unimproved grassland. The observation of preferential associations between fungi and host species contrasts with our results, but the incongruence may be explained by the different approaches used. In the study by Gollotte et al. (2004), soil was mixed and the two host plant species were reseeded into the plots, whereas the present study was conducted in an undisturbed system. Previous studies have demonstrated that soil disturbance may affect the infectivity of external hyphae of AM fungi (Jasper et al. 1991), and the procedure used by Gollotte et al. (2004) may therefore select for fungi that are less sensitive to soil disturbance and mainly propagate from spores. This excludes non-sporulating fungal species, which may depend on mycelial networks for their successful growth and propagation. The direct sampling strategy used in this study allowed for the assessment of such fungal species. Results support the hypothesis of Rosendahl and Stukenbrock (2004) that non-sporulating mycorrhizal species may be dominant in undisturbed soils where propagation by hyphal growth and the formation of large mycelial networks is favored. Mechanical mixing of soil, as in an agricultural soil, could on the other hand favor the spread and growth of fast-growing sporulating AM fungi.

The dominant phylogenetic cluster D was found in all plant species in all plots along the transect of the coastal grassland. It is not known if this cluster represents several clones, one clone or even one coherent mycelium. Rosendahl and Stukenbrock (2004) used a gene diversity analysis to analyse the genetic variation of the phylogenetic cluster D and suggested that the observed variation in the LSU rDNA sequences was most likely from one fungal individual. The dominant Glomus cluster found in this undisturbed vegetation could possibly represent a common mycelial network interconnecting the different plant species. The ability of AM fungi to form interconnecting mycelial networks between different plant species has been demonstrated under laboratory conditions by observing anastomoses between individual mycelia (Giovannetti et al. 2004). The findings of the present study support the hypothesis that interconnecting mycelia between different plant species also exist in natural vegetations. However, the genetic distance required to prevent anastomosis is not known (Jakobsen 2004), and it is important to evaluate if the genetic similarity found within the phylogenetic cluster D allows anastomosis and possibly the formation of a large mycelial network. Whether the resolution of the D2 region in the LSU rDNA is sufficient to reveal an individual mycelium is not known. Genotyping of AM fungi has so far only been possible using spores (Stukenbrock and Rosendahl 2004) or in vitro cultured fungi (Koch et al. 2004).

The ecological implications of interconnecting AM fungal hyphal networks for plant diversity and fitness are in- triguing. Grime et al. (1987) suggested that export of assimilates between donor and receiver plants through common mycelia networks could be an important element of the mechanism maintaining species-rich plant communities in infertile soils. More detailed sampling of roots from different plant species growing closely together in undisturbed vegetation could give access to more information about the extent of such mycelial networks. The common hyphal network may also allow some plants to obtain their inorganic nutrient at a low expense, by providing little or no carbon to the network (Jakobsen 2004). As soil disturbance may affect the community composition of AM fungi and the ability of the fungi to form mycelial networks between roots systems, these important attributes should be considered in future field studies of AM fungal communities and populations.

Acknowledgements We thank Lis Mathorne for technical assistance. This work was supported by grant 51-00-0373 from the Danish Natural Science Research Council (SNF). EHS was supported by Docent Scient, Dr. Lauritz Olsons Foundation.

\section{References}

Birch CPD (1986) Development of VA mycorrhizal infection in seedlings in semi-natural grassland turf. In: Gianinazzi-Pearson V, Gianinazzi S (eds) Physiological and genetical aspects of mycorrhizae. INRA, Paris, France, pp 233-237

Daniell TJ, Husband R, Fitter AH, Young JPW (2001) Molecular diversity of arbuscular mycorrhizal fungi colonising arable crops. FEMS Microbiol Ecol 36:203-209

Giovannetti M, Mosse B (1980) An evaluation of techniques for measuring vesicular arbuscular mycorrhizal infection in roots. New Phytol 84:489-500

Giovannetti M, Sbrana C, Strani P (2004) Patterns of below-ground plant interconnections established by means of arbuscular mycorrhizal networks. New Phytol 164:175-181

Gollotte A, van Tuinen D, Atkinson D (2004) Diversity of arbuscular mycorrhizal fungi colonising roots of the grass species Agrostis capillaries and Lolium perenne in a field experiment. Mycorrhiza 14:111-117

Grime JP, Mackey JML, Hillier SH, Read DJ (1987) Floristic diversity in a model system using experimental microcosms. Nature 328:420-422

Helgason T, Daniell TJ, Husband R, Fitter AH, Young JPW (1998) Ploughing up the wood-wide web? Nature 394:431

Helgason T, Merryweather JW, Dension J, Wilson P, Young JPW, Fitter AH (2002) Selectivity and functional diversity in arbuscular mycorrhizas of co-occurring fungi and plants from temperate deciduous woodland. J Ecol 90:371-384

Horton TR, Bruns TD (2001) The molecular revolution in ectomycorrhizal ecology: peeking into the black-box. Mol Ecol 10: $1855-1871$

Husband R, Herre EA, Young JPW (2002) Temporal variation in the arbuscular mycorrhizal communities colonizing seedlings in a tropical forest. FEMS Microbiol Ecol 42:131-136

Jakobsen I (2004) Hyphal fusion to plant species connections - giant mycelia and community nutrient flow. New Phytol 164:4-7

Jasper DA, Abbott LK, Robson AD (1991) The effect of soil disturbance on vesicular arbuscular mycorrhizal fungi in soils from different vegetation types. New Phytol 118:471-476

Kjøller R, Rosendahl S (2000) Detection of arbuscular mycorrhizal fungi (Glomales) in roots by nested PCR and SSCP (single stranded conformation polymorphism). Plant Soil 226:189-196 
Koch AM, Kuhn G, Fontanillas P, Fumagalli L, Goudet J, Sanders IR (2004) High genetic variability and low local diversity in arbuscular mycorrhizal fungal population. Proc Natl Acad Sci U S A 101:2369-2374

Kowalchuk GA, de Souza FA, van Veen J (2002) Community analysis of arbuscular mycorrhizal fungi associated with Ammophila arenaria in Dutch coastal sand dunes. Mol Ecol 11:571-581

Mosse B (1975) Specificity in VA mycorrhizas. In: Sanders FE, Mosse B, Tinker PB (eds) Endomycorrhizas. Academic, London, pp 409-484

Munkvold L, Kjøller R, Vestberg M, Rosendahl S, Jakobsen I (2004) High functional diversity within species of arbuscular mycorrhizal fungi. New Phytol 164:357-364

Nielsen KB, Kjøller R, Olsson PA, Schweiger PF, Andersen FØ, Rosendahl S (2004) Colonization intensity and molecular diversity of arbuscular mycorrhizal fungi in the aquatic plants Littorella uniflora and Lobelia dortmanna in Southern Sweden. Mycol Res 108:616-625

Phillips JM, Hayman DS (1970) Improved procedure for clearing and staining parasitic and vesicular-arbuscular mycorrhizal fungi for rapid assessment of infection. Trans Br Mycol Soc 55:158

Rosendahl S, Stukenbrock EH (2004) Community structure of arbuscular mycorrhizal fungi in undisturbed vegetation revealed by analyses of LSU rDNA sequences. Mol Ecol 13:3179-3186
Smith FA, Jakobsen I, Smith SE (2000) Spatial differences in acquisition of soil phosphate between two arbuscular mycorrhizal fungi in symbiosis with Medicago truncatula. New Phytol 147: 357-366

Stukenbrock EH, Rosendahl S (2004) Development and amplification of multiple co-dominant genetic markers from single spores of arbuscular mycorrhizal fungi by nested multiplex PCR. Fungal Genet Biol 42:73-80

Vandenkoornhuyse P, Husband R, Daniell TJ, Watson IJ, Ducke JM, Fitter AH, Young JPW (2002) Arbuscular mycorrhizal community composition associated with two plant species in a grassland ecosystem. Mol Ecol 11:1555-1564

van Tuinen D, Zhao B, Gianinazzi-Pearson V (1998a) PCR in studies of AM fungi: from primers to application. In: Varma A (ed) Mycorrhiza manual. Springer, Berlin Heidelberg New York, pp $387-400$

van Tuinen D, Jacquot E, Zhao B, Gollotte A, Gianinazzi-Pearson V (1998b) Characterization of root colonization profiles by a microcosm community of arbuscular mycorrhizal fungi using $25 \mathrm{~S}$ rDNA-target nested PCR. Mol Ecol 7:879-887 